\title{
A Secure Social Network Question and Answer System
}

\author{
M. Sai Sirisha ${ }^{1 *}$, S.S.S.N. Usha Devi $\mathbf{N}^{2}$ \\ ${ }^{1}$ Dept. of Computer Science and Engineering, University college of engineering JNTUK, Kakinada, India \\ ${ }^{2}$ Dept. of Computer Science and Engineering, University college of engineering JNTUK, Kakinada, India
}

Available online at: www.isroset.org

Received: 06/Oct/ 2018, Accepted: 14/Oct/ 2018, Online: 31/Oct/2018

\begin{abstract}
Now-a-days internet acts as a huge source of information whereas the Question and Answer (Q\&A) systems are widely used for information and knowledge sharing. The efficiency of the Question and Answer systems can be calculated depending on the factors like how correct the questions are answered within a short waiting time. For the user to be presented with correct answers for the questions asked, the questions are to be forwarded to those persons who are willing and capable to answer. This can be procured with the help of Question and Answer system with in a social network. This Secure Social Question and Answer system can be performed based on properties like common interest and mutual trust friendship among the people in the social network. The above two properties will be considered in identifying the appropriate answerer for a question. Security of the Question and Answer system is achieved with the use of bloom filter to protect user identities for the effective exchange of information.
\end{abstract}

Keywords-Question and Answer systems, Social networks, Bloom filter, Common interest.

\section{INTRODUCTION}

Information can be attained either by searching or asking. Every day a large amount of data is added in the internet from which searching the required information for the vast amount of data becomes a difficult task [1,2]. To avoid such cases of difficulties asking helps in getting the required information correctly within a short time, which can be achieved with the use of Question and Answer systems. These Social Question and Answer Systems mainly serve the purpose of getting information in case of non-factual questions like "what is use of Mars Orbital Mission?" To answer this type of questions the answerer needs to have knowledge in the particular field. Using this property Social Question and Answer system forwards the question to the one who is capable and willing to answer. This Q\&A system mainly involves two properties like common-interest between users and mutual trust friendship between the asker and answerer. We come across a lot of question and answer websites which are used by large number of Internet users. This Social Q\&A not only serves as a source for technical knowledge but also a source of receiving advice from those we trust. Thus this Social Q\&A serves both factual and nonfactual questions.

Every day a large number of questions are being posed in the internet. When a question is asked, there may be much number of users to answer the question. In such cases, the chance of answering the question is given only to those users who are capable and also willing to answer the question within a short time. In order to find out the most appropriate answerer, this Q\&A allows users to define tags i.e., users provide the interest categories to which their questions belong at the time asking questions. But there may be type of questions for which it is difficult to find the categories to some like "how to plan a perfect trip?"

As a result, there is a lot of demand for the social Q\&A systems which provide the most appropriate answer with least waiting time and having less number of unanswered questions.

The Social Q\&A not only meets the purpose of answering and asking questions but it should also protect the privacy of the users. Many users may ask or answer questions related to sensitive topics like health issues, political matters [7], in such cases the users need some privacy protection to avoid potential disclosure of personal information.

The Secure Social Question and Answer System is implemented with small number of users which depicts the real world implementation. The rest of the paper is organized as follows. Section II presents a concise review of

Literature survey. Sections III and IV present the details of the architecture of the Secure Social Q\&A and its enhancement methods. Section V measures the Social Q\&A's performance with various techniques used. Section VI and VII concludes the paper with the possible future works. 


\section{LITERATURE SURVEY}

The importance of Question and Answer systems are increasing day by day. The capability of the question and answer system depends on many factors like response time and the capability and willingness of the persons to respond for one's questions and correctness of the answer. The existing Q\&A systems with the asker-answerer relationship are different from the one which is present in the Social network Question and Answer system. Here in this, it concentrates on finding the appropriate answerer for the particular question. Social Network Q\&A also works well in case of opinion type questions.

There are many search engines which help to provide information when searched using keywords and also they provide a list of documents from which one has to search for the suitable answer, which is time consuming and also sometimes making it difficult to find the solution. In such situations Social Q\&A helps users to get answers for their question with in a less response time.

Some research categorizes questions into predefined categories, so that it makes the process of asking questions easier by choosing the category to which they belong. Also it helps the answerers to choose among the category with which they can comfortably answer questions.

Compared to previous Q\&A systems, Social Q\&A leverages both the common-interest and mutual-trust friendship to improve the performance of the Q\&A system. Different algorithms are used to determine user interest, question interest and question user mapping. Social Q\&A focuses on how to leverage social network properties in better identifying the capable answerer for a question with predefined interest categories.

\section{A SECURE Social NeTwORK QUESTION AND ANSWER SYSTEM}

\section{The Rationale of Social Q\&A Design}

Social network is formed assuming each person as a node and the connection between nodes is termed as a social relationship. An online social network connects friends with real-life relationship. Friends in an online social network tend to share similar interests and also trust each other. Using these two properties, Social Q\&A is developed incorporating social network to improve the quality of answers and also decrease the waiting time. Its main property is that it forwards questions to the social friends having common-interest and social closeness.

\section{The Design of Secure Social Q\&A}

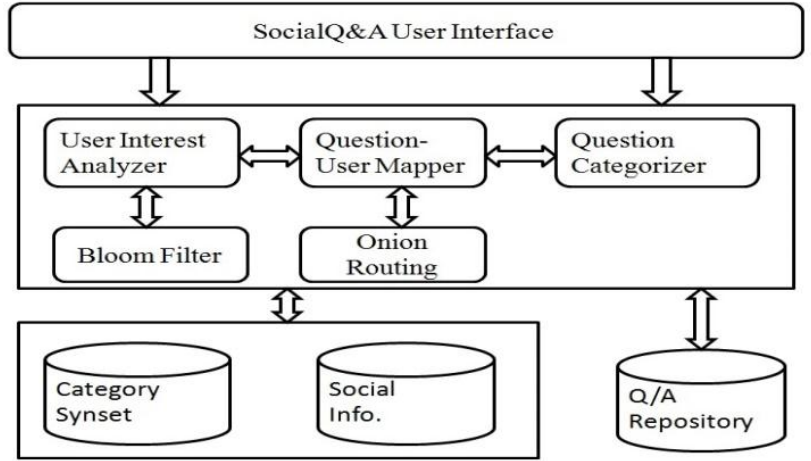

Figure1: The Architecture of Secure Social Q\&A.

Like all online social networks, it also has user's profiles that records user's information like interests, education, hobbies and etc. Users enter their information with the use of user interface which contains the details like users registration, login etc.

The architecture of Secure Social Q\&A defines the important components like User-Interest Analyzer, QuestionUser Mapper, Question Categorizer. Category Synset stores the information regarding the predefined categories and subcategories. Social Info. stores the data about the social activities of the users like searching friends, sending friend requests, accepting requests etc.

\section{User-Interest Analyzer}

The main purpose of the User-Interest Analyzer is that it uses the user's profile information and also user's interaction to analyze the interests of the users. At the time of registration users are allowed to define the topics in which they are interested in. Also if a user asks or answers a question in a particular category this shows that the user is interested in that particular category. Here, in this we have 6 predefined categories like Movies, Music, Software, Sports, News and Health.

\section{Algorithm 1: Pseudocode for User-Interest Analyzer}

Input: A user's profile, questions and answers

1: Parse the "interests" field to generate a token stream

2: Parse the "activities" field to generate a token stream

3: Use the inputs from the user's selection from the Music, Movie, Television and Book fields to generate token streams

4: for each token stream $T_{x}\left(T_{x}=T_{m u}, T_{m o}, T s, T_{n}\right)$ do

5: Check each token in the Synset

6: if a matching interest category Ii exists then

7: Update interest weight: $\mathrm{W}_{\mathrm{Ii}}++$

8: $\quad$ end if 
9: end for

10: Keep updating $\mathrm{W}_{\mathrm{Ii}}$ based on questions asked and answered and profile update.

11: Periodically update user's interest vector

The Algorithm 1 shows the psuedocode for User-Interest Analyzer which takes the users information and activities as the input. Using this algorithm every user is given an option of entering the category of their interests. Every interest category is assigned a weight $\mathrm{W}_{\mathrm{i}}$. Initially the weight of every interest category is considered as $0 . \mathrm{W}_{\mathrm{i}}$ is incremented by 1 for each appearance of the interest in the parsed information from a user's profile and interactions. The order of phrases does not necessarily represent the different preferences of a user. Thus, we count the frequency that an interest's synset appears in all phrases to indicate the user's preference on this interest, because the frequency represents a user's focus on an interest currently.

\section{Question Categorizer}

The primary task of Question Categorizer is to categorize a question into predefined interest categories based on the topic(s) of the question. We also allow users to input self-defined tags associate with questions, which are analysed in question parsing. Question Categorizer generates a vector of question Qi's interests, denoted by $\mathrm{V}_{\mathrm{Qi}}$, using a similar algorithm as Algorithm 1. While processing a question, Social Q\&A examines the tags and text of the question and generates a token string. The tokens are compared to Social Q\&A's Synset to determine the categories where the question belongs. We have calculated the interest weight without normalization in order to predict the user intelligence to answer a question of Interest.

\section{Question-User Mapper}

Question-User Mapper identifies the appropriate answerers for a given question. The potential answer providers are chosen from the asker's friends in the online social network. Note that the changes in a user's friends in the online social network do not affect the performance of Social Q\&A as it always uses a user's current friends. To check the appropriateness of a friend $\left(\mathrm{U}_{\mathrm{k}}\right)$ as an answer provider for a question, two parameters are considered: i) the interest similarity between the interest vectors of the friend and the question (denoted by $\mathrm{I} ; \mathrm{U}_{\mathrm{k}}$ ); and ii) the social closeness between the friend and the asker (denoted by $\mathrm{C} ; \mathrm{U}_{\mathrm{k}}$ ). The former represents the potential capability of a friend to answer the question, and the latter represents the willingness of a friend to answer the question.

Algorithm 2: Pseudocode for Question-User Mapper
Input: Interest vectors of a user, his/her friends and question

1: for each friend $U_{k}$ in the friend set of $U_{j}$ do

2: the similarity between their interest vectors

3: Compute asking and answering interaction frequency

4: Order the friends in descending order

5: Notify the top $\mathrm{N}$ friends

6: A list of potential answer providers.

\section{Disadvantages of the existing system}

Although Social Question and Answer system helps in finding appropriate answerers by forwarding questions to those persons who are capable and willing to answer a question. But there are certain cases in which the asker of the question may ask some questions which are related to some sensitive topics like political issues, health issues etc. In such cases there is a necessity to provide some privacy to the user who is asking the question and also the one who is going to answer the question. The privacy and security has to be provided to the users and also to the question from being known by the intruders.

\section{PROPOSED SYSTEM}

In the proposed system, in order to protect user privacy and to provide security to the questions techniques like Bloom Filter and Onion Routing are going to be used.

\section{Bloom Filter Based Personal Information Exchange}

To protect user privacy to a certain extent, friends should avoid exchanging such personal information directly. Instead, they should exchange the encrypted information of their friend lists and interest vectors. The challenge here is that the encrypted information should not only protect a user from revealing direct information to others but also serve counting the common friends and interests. The counting bloom filter technique can meet this requirement. Therefore, to handle this challenge, Social Q\&A uses the counting bloom filter technique to encrypt information that is exchanged between friends.

A counting bloom filter uses $\mathrm{K}$ hash functions to encrypt personal information for protection. The bloom filter results are stored in an integer array of $t$ entries. Each hash function encrypts the feed information into an integer $\mathrm{m}$ within $[0 ; t]$, and the $m^{\text {th }}$ entry of the integer array is increased 
by 1 . To search whether an information item is stored in a bloom filter, the information item is encrypted by each hash function of the bloom filter. If for each hashed result $\mathrm{m}$, the value at $\mathrm{m}^{\text {th }}$ entry in the array is larger than 0 , this information item has a higher probability of being stored in the bloom filter; otherwise, it is not stored in the bloom filter.

\section{Algorithm 3: Bloom Filter Algorithm}

\section{INPUT: USERS INFORMATION}

1: Bloom filter uses $\mathrm{K}$ hash functions to encrypt users information for protection.

2: Results are stored in an integer array of t entries.

3: Each hash function encrypts the feed information into an integer $\mathrm{m}$ within $[0 ; \mathrm{t}]$, and the mth entry of the integer array is increased by 1 .

4: If for each hashed result $\mathrm{m}$, the value at mth entry in the array is larger than 0 .

5: User's information item has a higher probability of being stored in the bloom filter.

6: Otherwise, it is not stored in the bloom filter.

7: Each user feeds each of his/her friend IDs into a bloom filter.

8: Friends exchange the bloom filter results instead of friendship information directly.

\section{Onion Routing Based Answer Forwarding}

Onion Routing is a technique for anonymous communication over a computer network. In an Onion network, messages are encapsulated in layers of encryption, analogous to layers of an onion. The encrypted data is transmitted through a series of network nodes called Onion Routers, each of which peels away a single layer, uncovering the data's next destination. When the final layer is decrypted, the message arrives at the destination.

Here in this network the use of Onion Routing comes into existence when a user asks a question and if the person who received the question is unable to answer the question he can forward the question to the other user's, in this case the one who is forwarding the question becomes the sender. Here the one who is receiving the question is only known that the sender of the question is the previous node only and is unaware of the initial sender and if there exists a case of further forwarding the question, it becomes the intermediate node. In Onion Routing, when a message is to be delivered in a path between the sender and the receiver there may exist many intermediate nodes in which every intermediate node is only known the address of its immediate neighbours only. So with the use of Onion Routing user's identity is unknown to the intermediate users who are forwarding the questions.

\section{Algorithm 4: Pseudocode for Onion Routing}

1: Identify the asker and the answerer.

2: Encrypt the identities of the asker at each layer.

3: Every intermediate node decrypts the previous layer and encrypts a new layer.

4: Repeat step 3 until it reaches the final node.

5: The final node decrypts the data.

\section{Advantages of the Proposed System}

Now-a-days in Social Networks protecting the identities and the personal information of the users is the challenging task ever. Using the techniques like Bloom Filter and Onion Routing measure are taken to protect user identities and also ensure secure connection while transferring data between the users.

\section{Experimental Results}

This Secure Social Q\&A project experiment is carried out among the M.Tech Computer Science students of Jawaharlal Technological University Kakinada. We created 27 users and assigned random interests to each and every user and made each student to ask and answer questions in predefined categories like Music, Movies, Sports, Research, Software etc.

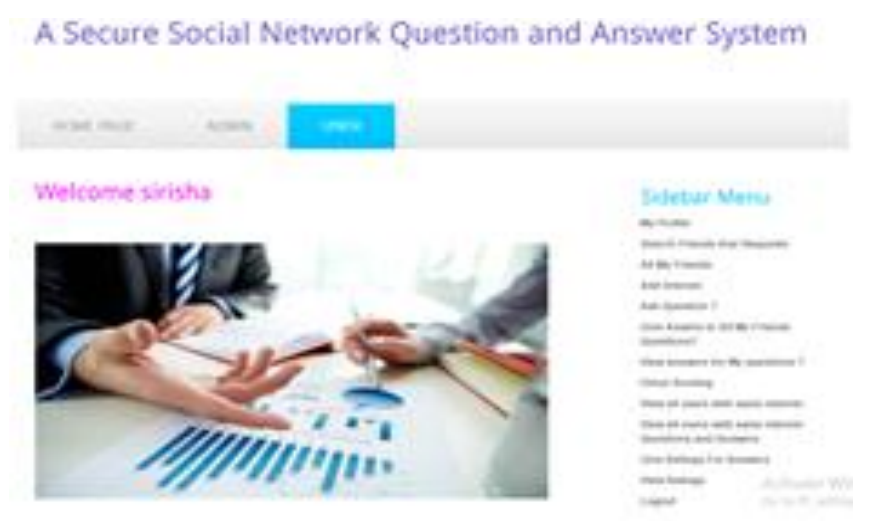

Figure 5.1: Prototype implementation of Secure Social Q\&A.

The above figure shows the implementation of the Secure Social Q\&A which provides certain options like viewing user's profile, adding interests, searching for friends and accepting friends. Through this portal a user can ask question and one can answer their friends questions. There is also an option to give ratings to the user's answers which helps in improving the quality of their answers. 


\section{A Secure Social Network Question and Answer System}

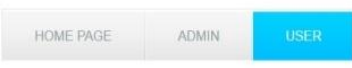

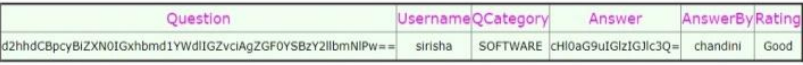

Back

Figure 5.2: Bloom Filter implementation of Secure Social Q\&A.

\section{A Secure Social Network Question and Answer System}

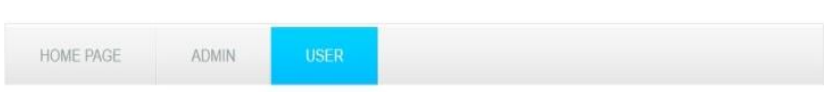

Onion Routing Based Questions And Answers

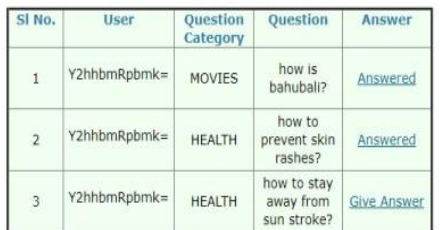

Figure 5.3: Onion Routing implementation of Secure Social Q\&A.

The purpose of using Bloom Filter is mainly to serve the purpose of hiding the question. This is mainly useful in case of people asking some sensitive questions that may be related to political issues, health issues in which user needs some privacy. So using Bloom Filter we are going to encrypt both the questions and answers. In the above figure 5.3 it is shown that the identities of the users who asked questions are encrypted this is because in Onion Routing when a message is passed in a path the intermediate nodes are unaware of the sender and the receiver of the message they just know the next node in the path to which the message has to be passed.

\section{Conclusion}

In present days Question and Answer systems become the part of our daily life, this Secure Social Q\&A system helps users to find answers for their question using two main properties like common-interest among the users and also mutual trust friendship. With the use of these properties Secure Social Q\&A helps users to find answers for their questions without spending more time using searching engines which produces a list of documents when searched and also the answerer also gets the questions of his interest without searching for the questions to answer.

\section{Future Work}

Comprehensive trace-driven experiments and analysis results on the real-world Q\&A activities from the Social Q\&A prototype show the promises of Social Q\&A to enhance answer quality and reduce answer wait time in current Q\&A systems, and demonstrate the secure and efficiency improvement achieved by the enhancements. Since same questions may be presented very differently and the same question may be answered differently in different situation, the scope for future work can be cooperated with other techniques like natural language processing and machine learning into Social Q\&A to find the redundant question with a large scale user set. Due to the dynamic of user behaviour, Social Q\&A can co-operate a machine learning method to adjust three parameters appropriately, which needs a large user base and much more usage.

\section{References}

[1] H. Shen, G. Liu, and N. Vithlani Socialq\&a: An online social network based question and answer system. In Proc. of ICCCN., vol. 3, Issue 1, March 2017

[2] B. Li, I. King, "Routing questions to appropriate answerers in community question answering services", Proc. 19th ACM Int. Conf. Inf. Knowl. Manage., pp. 1585-1588, 2010

[3] M. Richardson, R. White, "Supporting synchronous social Q\&A throughout the question lifecycle", Proc. 20th Int. Conf. World Wide Web, pp. 755-764, 2011.

[4] Z. Yang, J. Xue, C. Wilson, B. Y. Zhao, Y. Dai, "Uncovering user interaction dynamics in online social networks", Proc. 9th Int. AAAI Conf. Web Social Media, pp. 698-701, 2015.

[5] Z. Li, H. Shen, "Collective intelligence in the online social network of Yahoo!answers and its implications", Proc. 21st ACM Int. Conf. Inf. Knowl. Manage., pp. 455-464, 2012.

[6] J. Zhang, M. S. Ackerman, L. Adamic, "Expertise networks in online communities: Structure and algorithms", Proc. 16th Int. Conf. World Wide, pp. 221-230, 2007.

[7] J. Bian, Y. Liu, D. Zhou, E. Agichtein, H. Zha, "Learning to recognize reliable users and content in social media with coupled mutual reinforcement", Proc. 18th Int. Conf. World Wide Web, pp. 51-60, 2009.

[8] L. Hong, Z. Yang, B. D. Davison, "Incorporating participant reputation in community-driven question answering systems", Proc. Int. Conf. Comput. Sci. Eng., pp. 475-480, 2009.

[9] L. Hong, Z. Yang, B. D. Davison, "Incorporating participant reputation in community-driven question answering systems", Proc. Int. Conf. Comput. Sci. Eng., pp. 475-480, 2009.

[10] Y. R. Tausczik, J. W. Pennebaker, "Predicting the perceived quality of online mathematics contributions from users' reputations", Proc. SIGCHI Conf. Human Factors Comput. Syst., pp. 1885-1888, 2011.

[11] F. Liu, Y. Sun, B. Li, B. Li, X. Zhang, "FS2You: Peer-assisted semi-persistent online hosting at a large scale", IEEE Trans. Parallel Distrib. Syst., vol. 21, no. 10, pp. 1442-1457, Oct. 2010.

[12] A. Shtok, G. Dror, Y. Maarek, I. Szpektor, "Learning from the past: Answering new questions with past answers", Proc. 21st Int. Conf. World Wide Web, pp. 759-768, 2012.

[13] X. Quan, W. Liu, B. Qiu, "Term weighting schemes for question categorization", IEEE Trans. Pattern Anal. Mach. Intell., vol. 33, no. 5, pp. 1009-1021, May 2011.

[14] W. Song, W. Liu, N. Gu, X. Quan, T. Hao, "Automatic categorization of questions for user-interactive question 
answering", Inf. Process. Manage., vol. 47, pp. 147-156, 2011.

[15] B. Li, I. King, M. R. Lyu, "Question routing in community question answering: Putting category in its place", Proc. 20th ACM Int. Conf. Inf. Knowl. Manage., pp. 2041-2044, 2011.

[16] T. C. Zhou, M. R. Lyu, I. King, "A classification-based approach to question routing in community question answering", Proc. 21 st Int. Conf. World Wide Web, pp. 783-790, 2012.

[17] X. Cao, G. Cong, B. Cui, C. S. Jensen, C. Zhang, "The use of categorization information in language models for question retrieval", Proc. 18th ACM Conf. Inf. Knowl. Manage., pp. 265274, 2009.

[18] J. Guo, S. Xu, S. Bao, Y. Yu, "Tapping on the potential of Q\&A community by recommending answer providers", Proc. 17th ACM Conf. Inf. Knowl. Manage., pp. 921-930, 2008.

[19] L. Nie, Y. Zhao, X. Wang, J. Shen, T. Chua, "Learning to recommend descriptive tags for questions in social forums", ACM Trans. Inf. Syst., vol. 32, 2014.

\section{Authors' Profile}

Miss M. Sai Sirisha pursued Bachelor of Technology from Jawaharlal Nehru Technological University Vizianagram in the year 2016 and Master of Technology from Jawaharalal Nehru Technological University Kakinada in the year 2018.

Mrs. S.S.S.N. Usha Devi N. is currently working as an assistant professor in Jawaharlal Nehru Technological University Kakinada since 2013. 